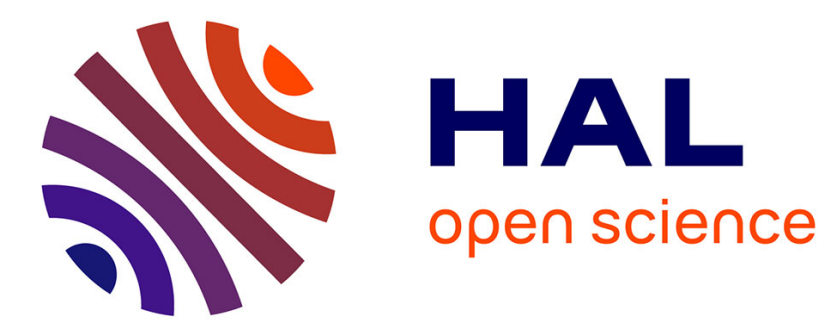

\title{
Chaotic behavior analysis based on sliding bifurcations
}

\author{
Djamila Benmerzouk, Jean-Pierre Null Barbot
}

\section{To cite this version:}

Djamila Benmerzouk, Jean-Pierre Null Barbot. Chaotic behavior analysis based on sliding bifurcations. Nonlinear Analysis: Hybrid Systems, 2010, 4 (3), pp.503-512. 10.1016/j.nahs.2009.12.001 . inria-00533447

\section{HAL Id: inria-00533447 https://hal.inria.fr/inria-00533447}

Submitted on 6 Nov 2010

HAL is a multi-disciplinary open access archive for the deposit and dissemination of scientific research documents, whether they are published or not. The documents may come from teaching and research institutions in France or abroad, or from public or private research centers.
L'archive ouverte pluridisciplinaire HAL, est destinée au dépôt et à la diffusion de documents scientifiques de niveau recherche, publiés ou non, émanant des établissements d'enseignement et de recherche français ou étrangers, des laboratoires publics ou privés. 


\title{
Chaotic behavior analysis based on sliding bifurcations
}

\author{
D. Benmerzouk ${ }^{1}$ and J-P Barbot ${ }^{2}$ \\ ${ }^{1}$ University of Tlemcen,Department of Mathematics,BP 119, 13000 Tlemcen, Algeria. \\ 2 ECS-Lab EA 3649, ENSEA, Cergy-Pontoise Paris, France, \\ \& EPI-ALIEN-INRIA.
}

\begin{abstract}
In this paper, a mathematical analysis of a possible way to chaos for bounded piecewise smooth systems of dimension 3 submitted to one of it's specific bifurcations, namely the sliding ones, is proposed. This study is based on period doubling method applied to the relied Poincaré maps.

Keywords:

Dynamic bifurcations (74H60), Chaotic behavior (74H65), Bifurcation of limits cycles and periodic orbits (37G15), Bifurcations connected with non transversal intersection (37G25).
\end{abstract}

\section{Introduction}

Piecewise smooth systems (p.w.s) considered in this paper are those defined by at least two sets of ordinary differential equations and switch from a smooth phase space to another one when some dynamic switching conditions occur, the sets that separate those phase spaces are smooth and of codimension one. Many special phenomena are relied to those systems (in addition to the classic ones) particularly the sliding ones; recall that those phenomena are well-known and studied since at least the famous Filippov book [13]. Moreover, the Russian school has also proposed pioneer works in piecewise smooth bifurcations $[11,12]$. More recently, Mario di Bernardo and coauthors gave a bifurcation's classifications for piecewise smooth systems $[8,6,9]$ using the relied Poincaré maps. On the basis of this special classifications, we analyze the possibility of generating chaos for bounded p.w.s systems via these kinds of bifurcations; we have already presented some results in $[2,1]$ concerning respectively the grazing bifurcation's and the corner bifurcation's cases. Hereafter, we investigate the possibility of generating chaos for bounded p.w.s systems of dimension 3 submitted to sliding bifurcations. Obviously, this kind of piecewise smooth systems and corresponding bifurcation's analysis already exists $[16,15]$, nevertheless, at our knowledge, a general mathematical study of a way to chaos via this type of bifurcations has never been proposed. Consequently, in this paper, following the analysis given in $[4,3]$ and based on Lyapounov-Schmidt method, a solution analysis is proposed for generating chaotic behavior, this will be done using period doubling method. 
The paper is organized as follows: In section II, the problem statement is established; In section III , the problem analysis is developed, a way to chaos is proposed in section IV and an academic example based on this approach with simulations results are presented in section $\mathrm{V}$.

\section{Recalls and Problem statement}

Let us consider the following piecewise smooth system:

$$
\dot{x}=\left\{\begin{array}{l}
F_{1}(x) \text { if } H(x) \geq 0 \\
F_{2}(x) \text { if } H(x)<0
\end{array}\right.
$$

where $x: I \longrightarrow D, D$ is an open bounded domain of $R^{3}, I \subset R^{+}$, generally $I$ is the time interval.

$$
F_{1}, F_{2}: C^{1}(I, D) \longrightarrow C^{k}(I, D), \quad \text { for } k \geq 4
$$

where $C^{k}(I, D)$ is the set of $C^{k}$ functions defined on $I$ and having values in $R^{3}$, the norm for $C^{k}(I, D)$ is defined as follows:

$x \in C^{k}(I, D) \quad:\|x\|=\sup _{t \in I}\|x(t)\|_{e}+\sup _{t \in I}\|\dot{x}(t)\|_{e}+\ldots+\sup _{t \in I}\left\|x^{(k)}(t)\right\|_{e}$

where $x^{(k)}($.$) denotes the k^{t h}$ derivative of $x($.$) and \|.\|_{e}$ is a norm defined on $R^{3}$.

According to [5], $\left(C^{k}(I, D),\|\cdot\|\right)$ is a Banach space.

Thus, both vector fields $F_{1}$ and $F_{2}$ are sufficiently regular on their domains of definitions such that the flows $\Phi_{i}, i=1,2$ generated by each vector field $F_{i}$ are defined as the operators that satisfy:

$\frac{\partial \Phi_{i}(x, t)}{\partial t}=F_{i}\left(\Phi_{i}(x, t)\right)$ and $\Phi_{i}(x, 0)=x, i=1,2$.

$H: D \longrightarrow R$ is a $C^{1}$ application, it is a phase space boundary between both regions of smooth dynamics, $H$ defines the set:

$S=\{x(t) \in D: H(x(t))=0\}$

and $S$ is the switching manifold and divides the phase space into two regions:

$S^{+}=\{x(t) \in D: H(x(t))>0\}$

$S^{-}=\{x(t) \in D: H(x(t))<0\}$

Moreover for being in the context of sliding phenomena, it is assumed that there exists a subset of switching manifold $\bar{S} \subset S$ denoted as sliding region which is simultaneously attracting from $S^{+}$and $S^{-}$, so, considering any neighborhood $v_{\bar{S}}$ of $\bar{S}$, the existence of $\bar{S}$ is characterized by the following hypothesis:

$\left.\mathrm{H}_{1}\right)<\nabla H(x(t)), F_{2}(x(t))-F_{1}(x(t))>\in R_{+}^{*}$, for all $x(t) \in v_{\bar{S}}$.

where $<., .>$ is a usual scalar product on $R^{3}$.

Thus, under $\mathrm{H}_{1}$ ), if the system trajectory crosses $\bar{S}$, the sliding motion evolves in $\bar{S}$ until it eventually reaches its boundary, this motion can also be described by considering an appropriate vector $F_{\bar{S}}$ witch lies within the convex hull of $F_{1}$ and $F_{2}$ and is tangent to $\bar{S}$ for each $x(t) \in v_{\bar{S}}, F_{\bar{S}}$ is given by: 


$$
F_{\bar{S}}(x(t))=\frac{F_{1}(x(t))+F_{2}(x(t))}{2}+H_{u}(x(t)) \frac{F_{1}(x(t))-F_{2}(x(t))}{2},
$$

where $H_{u}: D \longrightarrow R$ is deduced from the equivalent vector method [13, 17], in fact $H_{u}$ is given using the tangential condition of $F_{\bar{S}}$ on the switching manifold i.e. $\left\langle\nabla H, F_{\bar{S}}>=0\right.$ which implies that :

$$
H_{u}(x(t)):=-\frac{<\nabla H(x(t)), F_{1}(x(t))+F_{2}(x(t))>}{<\nabla H(x(t)), F_{1}(x(t))-F_{2}(x(t))>}
$$

and

$$
-1 \leq H_{u}(x(t)) \leq 1, \text { for } x(t) \in v_{\bar{S}}
$$

So, the sliding region is redefined as :

$$
S_{v}=\left\{x(t) \in v_{\bar{S}}:-1 \leq H_{u}(x) \leq 1\right\}
$$

It's follows from $\mathrm{H}_{1}$ ) and (4) that sliding phenomena is characterized by:

$$
<\nabla H(x(t)), F_{2}(x(t))>>0><\nabla H(x(t)), F_{1}(x(t))>\quad \text { for } x(t) \in v_{\bar{S}}
$$

and the boundary of the sliding region is defined as:

$$
\partial S_{v}^{+}=\left\{x(t) \epsilon S_{v}: H_{u}(x(t)=1\} \text { and } \partial S_{v}^{-}=\left\{x(t) \epsilon S_{v}: H_{u}(x(t)=-1\} .\right.\right.
$$

The system (1) characterizes the behavior of an increasing number of dynamic systems particularly in Applied Sciences and Engineering, those piecewise smooth systems can present a specific switching bifurcations particularly when depending on some parameters, thus in order to present a complete sliding bifurcation analysis, it is assumed that the system (1) is bounded and depends smoothly on a parameter $\varepsilon$ such that at $\varepsilon=0$, there exists a periodic orbit $\bar{x}(t)$ that slides at the point $\bar{x}$ corresponding to $\bar{t}$ (where $\bar{t}$ is defined modulo the periodicity of (1)) and there is no other sliding point along this orbit other than the point $\bar{x}$ and this for a sufficiently small neighborhood of $\varepsilon$.

Consequently, the system (1) is rewrite as follows:

$$
\dot{x}=\left\{\begin{array}{l}
F_{1}(x, \varepsilon) \text { if } H(x) \geq 0 \\
F_{2}(x, \varepsilon) \text { if } H(x)<0
\end{array}\right.
$$

and it is assumed that there exist a neighborhood $v_{\varepsilon}$ of $\varepsilon=0$, a neighborhood $v_{x}$ of $x=\bar{x}$ such that the following hypotheses are verified at this bifurcation point $(\bar{x}, \bar{t})$ :

$\left.\mathrm{H}_{2}\right) H(\bar{x})=0$ and $\nabla H(\bar{x}) \neq 0$.

$\left.\mathrm{H}_{3}\right) H_{u}(\bar{x})=-1 \Leftrightarrow<\nabla H(\bar{x}), F_{1}(\bar{x})>=0$

The assumption $\mathrm{H}_{2}$ ) means that the bifurcation point belongs to the switching manifold and its neighborhood is correctly defined ( $\bar{x}$ is isolated) and the assumption $\mathrm{H}_{3}$ ) means that $\bar{x}$ is located on the boundary of the sliding region. 
Without loss of generality (thanks to a coordinate change), the bifurcation point is assumed to be located at $(\bar{x}, \bar{t})=(0,0)$.

According to [6], four possible cases of bifurcations involving sliding are possible:

Case 1: Sliding bifurcation of type 1: here, the sliding flow is assumed to move locally towards the boundary of the sliding region when it is perturbed from bifurcation point (see fig 1):

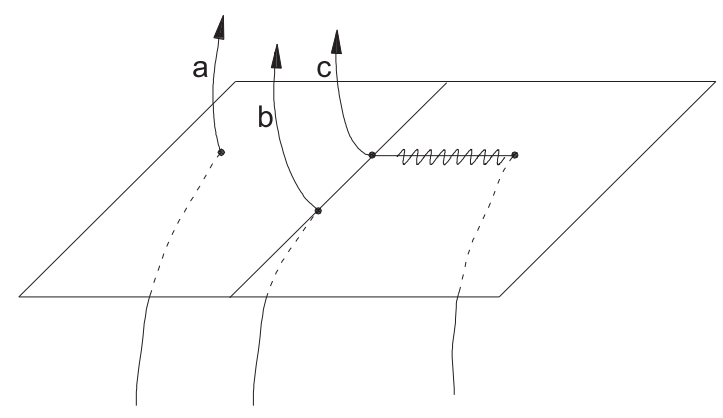

Figure 1: Sliding bifurcation of type 1

This yields the following assumption:

$\left.\mathrm{A}_{1}\right) \quad \frac{\partial^{2} H\left(\phi_{1}(0,0)\right)}{\partial t^{2}}=\left\langle\nabla H(0), \frac{\partial F_{1}(0,0)}{\partial x} F_{1}(0,0)\right\rangle>0$.

The determination of the Poincaré map is based on the determination of the Zero Discontinuity Map (noted ZDM), this map captures all the informations concerning the switching trajectories and is given by:

$$
Z D M_{1}(x, \varepsilon)= \begin{cases}\varepsilon x & \text { if }<\nabla H, x>\leq 0 \\ \varepsilon x+\varepsilon^{2} v_{1}(x)+o\left(\varepsilon^{3}\right) & \text { if }<\nabla H, x>>0\end{cases}
$$

where:

$$
v_{1}(x)=\frac{1}{2} \frac{<\nabla H, \frac{\partial F_{1}}{\partial x} x>^{2}}{<\nabla H, F_{2}><\nabla H, \frac{\partial F_{1}}{\partial x} F_{1}>}\left(F_{2}-F_{1}\right)
$$

$o\left(\varepsilon^{\alpha}\right) \longrightarrow 0$ when $\varepsilon \longrightarrow 0, \alpha \in R^{+}$and for the sake of compactness, the values of functions $F_{1}, F_{2}, \frac{\partial F_{1}}{\partial x}$ and $\nabla H$, defined in $v_{1}$, stand (and also for the values of the functions given in the coefficients $v_{2}, v_{3}, v_{4}$ of the next cases) respectively for $F_{1}(0,0), F_{2}(0,0), \frac{\partial F_{1}}{\partial x}(0,0)$ and $\nabla H(0)$. Moreover, the projection of $x$ on the Poincaré map is also noted by $x$.

Case 2: Grazing sliding bifurcation: here, the sliding flow moves towards the edge of the sliding strip and the sliding trajectory tends to leave $S$ (see fig $2)$. 


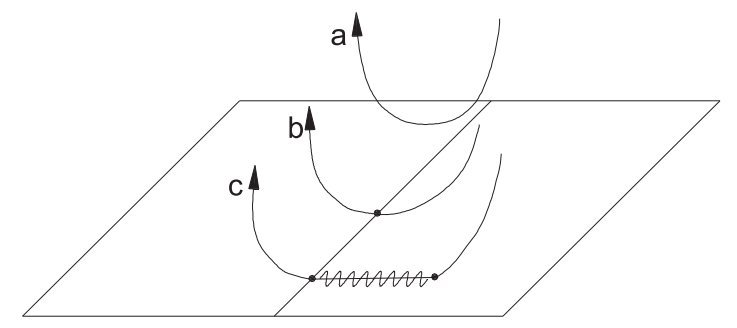

Figure 2: Grazing Sliding bifurcation

This yields the following assumption:

$\left.\mathrm{A}_{2}\right) \quad \frac{\partial^{2} H\left(\phi_{2}(0,0)\right)}{\partial t^{2}}=\left\langle\nabla H(0), \frac{\partial F_{2}(0,0)}{\partial x} F_{2}(0,0)\right\rangle>0$.

and the corresponding ZDM is given by:

$$
Z D M_{2}(x, \varepsilon)= \begin{cases}\varepsilon x & \text { if }<\nabla H, x>\geq 0 \\ \varepsilon x+\varepsilon v_{2}(x)+o\left(\varepsilon^{\frac{3}{2}}\right) & \text { if }<\nabla H, x><0\end{cases}
$$

where:

$$
v_{2}(x)=-\frac{<\nabla H, x>}{<\nabla H, F_{2}>}\left(F_{2}-F_{1}\right)
$$

Case 3: Sliding bifurcation of type 2 (or switching-sliding bifurcation): here, contrary to the conditions requested for the two previous cases, the vector field $F_{\bar{S}}$ must point away from the boundary of the sliding region at the bifurcation point and after a sliding occurs (see fig 3 ):

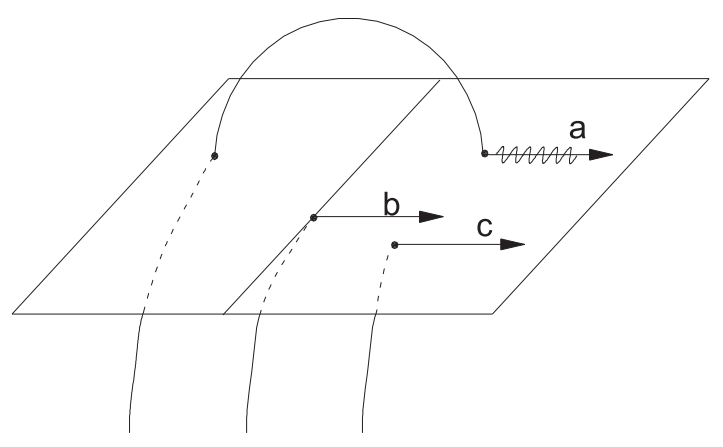

Figure 3: Sliding bifurcation of type 2 
Thus knowing that along $\partial \bar{S}: F_{\bar{S}}=F_{1}$, the following assumption is required: A $) \quad \frac{\partial^{2} H\left(\phi_{1}(0,0)\right)}{\partial t^{2}}=\left\langle\nabla H(0), \frac{\partial F_{1}(0,0)}{\partial x} F_{1}(0,0)\right\rangle<0$.

and the corresponding ZDM is given by:

$$
Z D M_{3}(x, \varepsilon)= \begin{cases}\varepsilon x & \text { if }<\nabla H, x>\leq 0 \\ \varepsilon x+\varepsilon^{3} v_{3}(x)+o\left(\varepsilon^{4}\right) & \text { if }<\nabla H, x>>0\end{cases}
$$

where :

$$
\begin{aligned}
v_{3}(x)= & \frac{2}{3} \frac{<\nabla H, \frac{\partial F_{1}}{\partial x} x>^{3}}{<\nabla H, F_{2}><\nabla H, \frac{\partial F_{1}}{\partial x} F_{1}>^{2}} \\
& \left(\frac{\partial F_{1}}{\partial x}+\frac{I_{d}}{<\nabla H, F_{2}>}\left(<\nabla H, \frac{\partial F_{1}}{\partial x} F_{1}-\frac{\partial F_{1}}{\partial x} F_{2}>\right)\right)\left(F_{2}-F_{1}\right)
\end{aligned}
$$

and $I_{d}$ denotes the identity matrix of appropriate dimension.

Case 4: Multisliding bifurcation: here the sliding flow is tangential to the boundary of the sliding strip at the bifurcation point and for further variation of the bifurcation parameter the trajectory may leave completely the manifold $S$ going to $S^{+}$(see fig 4 ):

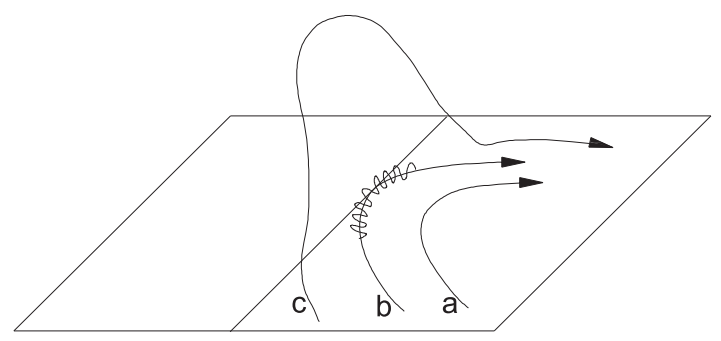

Figure 4: Multisliding bifurcation

So the following assumption is required:

$$
\left.\mathrm{A}_{4}\right) \quad \frac{\partial^{3} H\left(\phi_{1}(0,0)\right)}{\partial t^{3}}=\left\langle\nabla H(0),\left(\frac{\partial F_{1}(0,0)}{\partial x}\right)^{2} F_{1}(0,0)\right\rangle<0 .
$$

and the corresponding ZDM is given by:

$$
Z D M_{4}(x, \varepsilon)= \begin{cases}\varepsilon x & \text { if }<\nabla H, x>\geq 0 \\ \varepsilon x+\varepsilon^{2} v_{4}(x)+o\left(\varepsilon^{\frac{5}{2}}\right) & \text { if }<\nabla H, x><0\end{cases}
$$

where: 
$v_{4}(x)=-\frac{9}{2} \frac{<\nabla H, \frac{\partial F_{1}}{\partial x} x>^{2}}{<\nabla H, F_{2}><\nabla H,\left(\frac{\partial F_{1}}{\partial x}\right)^{2} x>}\left(\frac{\partial F_{1}}{\partial x}-\frac{<\nabla H, \frac{\partial F_{1}}{\partial x} F_{2}>}{<\nabla H, F_{2}>} I_{d}\right)\left(F_{2}-F_{1}\right)$

For each type of sliding, the corresponding Poincaré map (noted $P_{i}$ ) is obtained by composing the corresponding ZDM with the time T-flow periodic map associated to the system and given by:

$$
\Pi_{p e r}: x \rightarrow P x+Q \varepsilon+o(\|x\|, \varepsilon)
$$

where $P$ is a nonsingular matrix and $Q$ is a non null vector of appropriate dimensions.

\section{Problem analysis}

In this section, the existence and uniqueness of periodic solution for the system (6) may be discussed for the four cases of slidings .

First, recall that a fixed point of $P_{i}(., \varepsilon)$ represents an $m T$ periodic orbit, where $m$ is a natural number and $T$ is the period, therefore searching a periodic solution of (6) is equivalent to resolve for each case of sliding $i=1,2,3$, 4 , the following equations:

$$
P_{i}(x, \varepsilon)=x
$$

So, two possibilities appear:crossing and non crossing cases,

First possibility: there is no crossing i.e. $\left(\langle\nabla H, x\rangle \in R_{-}\right.$for case 1 and $3)$ or $(<\nabla H, x\rangle \in R_{+}$for case 2 and 4$)$. Thus, one only considers the first equation of the systems (7), (9), (11), (13) composed with the periodic map (15) and the problem becomes to analyze the same equation for the four cases given by:

$$
\gamma(x, \varepsilon):=P(\varepsilon x)+Q \varepsilon-x=0
$$

Thus, for sufficiently small $\varepsilon$ in the neighborhood $v_{\varepsilon}$ of 0 , the Implicit Functions theorem gives:

Lemma 1 Under conditions $\left.H_{j}\right), j=1,2,3$, and assumptions $A_{i}$ ) specific to each case $i=1,2,3,4$, there exists a neighborhood $v_{\varepsilon=0} \subset v_{\varepsilon}$ in $R, a$ neighborhood $\nu_{x=0}$ on the Poincaré section's (defined in $R^{2}$ ) and an unique application $x^{*}: v_{\varepsilon=0} \longrightarrow \nu_{x=0}$ solution of $\gamma\left(x^{*}(\varepsilon), \varepsilon\right)=0$ such that $x^{*}(0)=0$. Furthermore, the periodic solution of (6), $x^{*}$ depends continuously on $\varepsilon$.

Second possibility: a crossing occurs i.e. $(<\nabla H, x\rangle \in R_{+}^{*}$ for case 1 and 3$)$ or $\left(<\nabla H, x>\in R_{-}^{*}\right.$ for case 2 and 4$)$ :

Thus the problem of finding a periodic solution of (6) is equivalent to analyze for each case $i=1,2,3,4$ the following corresponding equations:

$$
P_{i}(x, \varepsilon)-x=0
$$

where : 
- For case 1:

$$
P_{1}(x, \varepsilon)=P\left(\varepsilon x+\varepsilon^{2} v_{1}(x)\right)+Q \varepsilon+o\left(\varepsilon^{3}\right)
$$

where $v_{1}(x)$ is given in (8) and $\varepsilon \in v_{\varepsilon}$.

- For case 2:

$$
P_{2}(x, \varepsilon)=P\left(\varepsilon x+\varepsilon v_{2}(x)\right)+Q \varepsilon+o\left(\varepsilon^{\frac{3}{2}}\right)
$$

where $v_{2}(x)$ is given in $(10)$ and $\varepsilon \in v_{\varepsilon}$.

- For case 3:

$$
P_{3}(x, \varepsilon)=P\left(\varepsilon x+\varepsilon^{3} v_{3}(x)\right)+Q \varepsilon+o\left(\varepsilon^{4}\right)
$$

where $v_{3}(x)$ is given in $(12)$ and $\varepsilon \in v_{\varepsilon}$.

- For case 4:

$$
P_{4}(x, \varepsilon)=P\left(\varepsilon x+\varepsilon^{2} v_{4}(x)\right)+Q \varepsilon+o\left(\varepsilon^{\frac{5}{2}}\right)
$$

where $v_{4}(x)$ is given in $(14)$ and $\varepsilon \in v_{\varepsilon}$.

The analysis of equations (18) is equivalent to analyze the following ones:

$$
\beta_{i}(x, \varepsilon):=P_{i}(x, \varepsilon)-x=0, \quad i=1,2,3,4
$$

and the Implicit Functions theorem gives:

Lemma 2 Under conditions $\left.H_{j}\right), j=1,2,3$, and assumptions $\left.A_{i}\right)$ specific to each case of sliding bifurcations $i=1,2,3,4$, there exists a neighborhood $v_{\varepsilon=0}^{i} \subset$ $v_{\varepsilon}$ in $R$, a neighborhood $\nu_{x=0}^{i}$ on the Poincaré section's (defined in $R^{2}$ ) and an unique application $x_{i}^{* *}: v_{\varepsilon=0}^{i} \longrightarrow \nu_{x=0}^{i}$ solution of $\beta_{i}\left(x_{i}^{* *}(\varepsilon), \varepsilon\right)=0$ such that $x_{i}^{* *}(0)=0$. Furthermore, the periodic solution of $(6), x^{* *}$ depends continuously on $\varepsilon$.

\section{Way to Chaos}

It is well known that a deterministic chaotic system is characterized by a great sensitivity to the initial conditions, a bounded behavior and "the strange"structure of its attractor. Moreover, according to [14] or [18], one way to generate chaotic behavior for one dimensional discrete systems is those based on period doubling method. In this section, we propose a mathematical analysis for bounded p.w.s systems of dimension 3, the associated Poincaré map is thus of dimension 2 , thus at this step, the famous result of Lie and Yorke [18] : "Period three implies chaos" is not available but here the most important mathematical tool used is the Implicit Function Theorem, this enables us to construct not an "ordinary" solution but a "branch" of solutions depending continuously on the parameter $\varepsilon$ and as the Poincaré map is chosen to be orthogonal to the sliding surface, at $\varepsilon=0$ the considered system will be defined in dimension 1 and this enables us to invoke this result. More precisely, 
the problem becomes to determine for each map $P_{i}, i=1,2,3,4$, three distinct points $x_{i}, y_{i}$ and $z_{i}$ such that: $P_{i}\left(x_{i}, \varepsilon\right)=y_{i}, P_{i}\left(y_{i}, \varepsilon\right)=z_{i}$ and $P_{i}\left(z_{i}, \varepsilon\right)=x_{i}$. This well be done naturally in three steps as follows:

First step: analysis of the equation:

$$
P_{i}\left(x_{i}, \varepsilon\right)=y_{i}=x_{i}+\eta_{i}
$$

where for a sake of simplicity, $\eta_{i}$ stands for a vector defined in $R^{2}$, having only one component equal to some fixed value (noted also $\eta_{i}$ ) and the other component is zero.

Thus, the equation (24) is equivalent to:

$$
\Psi_{i}\left(x_{i}, \varepsilon, \eta_{i}\right):=P_{i}\left(x_{i}, \varepsilon\right)-x_{i}-\eta_{i}=0
$$

and the next lemma is obtained with the same arguments than the previous one, note that the assumption needed at this step is that $\varepsilon$ must be very small relatively to 1 which is obvious and thus:

Lemma 3 Under conditions $\left.H_{j}\right), j=1,2,3$ and $A_{i}$ ) specific to each case $i=$ $1,2,3,4$, there exists a neighborhood $\vartheta_{\varepsilon=0}^{i}$ in $R$, a neighborhood $v_{\eta_{i}=0}$ in $R, a$ neighborhood $v_{x_{i}=0}$ on the Poincaré section's (defined in $R^{2}$ ) and an unique application $x_{i}^{*}: \vartheta_{\varepsilon=0}^{i} \times v_{\eta_{i=0}} \longrightarrow v_{x_{i}=0}$ solution of $\Psi_{i}\left(x_{i}^{*}\left(\varepsilon, \eta_{i}\right), \varepsilon, \eta_{i}\right)=0$ such that $x_{i}^{*}(0,0)=0$. Furthermore, $x_{i}^{*}$ depends continuously on $\varepsilon$ and $\eta_{i}$.

Second step: analysis of the equation:

$$
P_{i}\left(P_{i}\left(x_{i}, \varepsilon\right), \varepsilon\right)=z_{i}=y_{i}+\mu_{i}=x_{i}^{*}\left(\varepsilon, \eta_{i}\right)+\eta_{i}+\mu_{i}
$$

where $\mu_{i}$ stands for a vector defined on $R^{2}$, having only one component equal to some fixed value(noted also $\mu_{i}$ ) and the other is null. Thus, equation (26) is equivalent to:

$$
\Gamma_{i}\left(\varepsilon, \eta_{i}, \mu_{i}\right):=P_{i}\left(x_{i}^{*}\left(\varepsilon, \eta_{i}\right)+\eta_{i}, \varepsilon\right)-x_{i}^{*}\left(\varepsilon, \eta_{i}\right)-\eta_{i}-\mu_{i}=0
$$

and in order to continue the process, the following hypothesis according to each case is necessary:

$$
\left.\mathrm{H}_{1}^{i}\right)^{\prime} \quad \frac{\partial \Gamma_{i}}{\partial \eta_{i}}(0,0,0) \neq 0 .
$$

It comes:

Lemma 4 Under conditions $\left.\left.H_{j}\right), j=1,2,3, A_{i}\right)$ specific to each case $i=$ $1,2,3,4$ and $H_{1}^{i}$ )', $i=1,2,3,4$ specific to each case, there exists a neighborhood $v_{\varepsilon=0}^{i} \subset \vartheta_{\varepsilon=0}^{i}$, a neighborhood $\nu_{\eta_{i=0}} \subset v_{\eta_{i=0}}$, a neighborhood $\nu_{\mu_{i}=0}$ in $R$ and an unique application $\eta_{i}^{*}: v_{\varepsilon=0}^{i} \times \nu_{\mu_{i}=0} \longrightarrow \nu_{\eta_{i=0}}$ solution of $\Gamma_{i}\left(\varepsilon, \eta_{i}^{*}\left(\varepsilon, \mu_{i}\right), \mu_{i}\right)=0$ such that $\eta_{i}^{*}(0,0)=0$. Furthermore, $\eta_{i}^{*}$ depends continuously on $\varepsilon$ and $\mu_{i}$.

Third step: analysis of the equation:

$$
P_{i}\left(P_{i}\left(P_{i}\left(x_{i}, \varepsilon\right), \varepsilon\right), \varepsilon\right)=x_{i}
$$


The equation (27) is equivalent to:

$$
\Pi_{i}\left(\varepsilon, \mu_{i}\right):=P_{i}\left(x^{*}\left(\varepsilon, \eta_{i}^{*}\left(\varepsilon, \mu_{i}\right)\right)+\eta_{i}^{*}\left(\varepsilon, \mu_{i}\right)+\mu_{i}, \varepsilon\right)-x_{i}^{*}\left(\varepsilon, \eta_{i}^{*}\left(\varepsilon, \mu_{i}\right)\right)=0
$$

So the following hypothesis is required for each case $i=1,2,3,4$ :

$$
\left.\mathrm{H}_{2}^{i}\right)^{\prime} \quad \frac{\partial \Pi_{i}}{\partial \mu_{i}}(0,0) \neq 0
$$

and one obtains:

Lemma 5 Under conditions $\left.\left.H_{j}\right), j=1,2,3, A_{i}\right), i=1,2,3,4$ specific to each case and $\left.H_{1}^{i}\right)^{\prime}, H_{2}^{i}$ )', $i=1,2,3,4$ specific to each case, there exists a neighborhood $\omega_{\varepsilon=0}^{i} \subset v_{\varepsilon=0}^{i}$, a neighborhood $\theta_{\mu_{i=0}}$ in $\nu_{\mu_{i=0}}$ and an unique application $\mu_{i}^{*}$ : $\omega_{\varepsilon=0}^{i} \longrightarrow \theta_{\mu_{i=0}}$ solution of $\Pi_{i}\left(\varepsilon, \mu_{i}^{*}(\varepsilon)\right)=0$ such that $\mu_{i}^{*}(0)=0$. Furthermore, $\mu_{i}^{*}$ depends continuously on $\varepsilon$.

Remark 1 Assumptions noted $H_{j}$ ) are relied to the sliding phenomena and are so all necessary but the assumptions noted $A_{i}$ ) are relied to each of the four types of sliding bifurcations and assumption noted $H_{k}^{i}$ )', $k=1,2$ are relied to chaotification processus corresponding to each case of slidings $i=1,2,3,4$.

The next corollary sums up the previous results:

Corollary 6 Under conditions $\left.\left.H_{j}\right), j=1,2,3, A_{i}\right), i=1,2,3,4$ specific to each case and $\left.\left.H_{1}^{i}\right)^{\prime}, H_{2}^{i}\right)^{\prime}, i=1,2,3,4$ specific to each case, the bounded system (6), of dimension 3 , admits a chaotic behavior.

Remark 2 A chaotic behavior in the sense of Lie and Yorke is not generally guarantied for systems of dimension greater than 3 because the period doubling as a way to chaos, at the best of our knowledge, is only proved for one dimensional discrete systems. Nevertheless, a period doubling is an indication for an eventual chaotic behavior. Moreover, the constraint ${ }^{1} n-d=2$ is not so restrictive: for switched system (6) with sliding manifold separating the state space in two parts, condition $n-d=2$ is verified. Thus the period doubling is also valid in this case and the previous corollary may be rewritten with condition $n-d=2$ instead of system dimension equal to 3.

Comparing this result to the previous one given in $[2,1]$ corresponding respectively to the grazing bifurcation and corner bifurcation highlights that sliding bifurcations need less restrictive conditions, consequently, sliding bifurcations seem better choice for many applications as chaotic emitter design.

\section{An illustrative example}

In this section, one illustrates the proposed approach using an academic example submitted to sliding bifurcation of type 2, nevertheless, the other types of sliding

\footnotetext{
${ }^{1} n-1$ is the dimension of the relied Poincaré map (in our case $n-1=2$ ) and $d$ is the bifurcation codimension.
} 
bifurcations can also be treated in the same way.

So, let us consider the following piecewise smooth system:

$$
\begin{aligned}
\dot{x}_{1} & =f_{11}\left(x_{1}, x_{2}, x_{3}\right) \\
\dot{x}_{2} & =f_{12}\left(x_{1}, x_{2}, x_{3}\right) \quad \text { for } \quad x_{2} \leq 0 \\
\dot{x}_{3} & =f_{13}\left(x_{1}, x_{2}, x_{3}\right) \\
\text { and } & \\
\dot{x}_{1} & =f_{21}\left(x_{1}, x_{2}, x_{3}\right) \\
\dot{x}_{2} & =f_{22}\left(x_{1}, x_{2}, x_{3}\right) \quad \text { for } \quad x_{2}>0 \\
\dot{x}_{3} & =f_{23}\left(x_{1}, x_{2}, x_{3}\right)
\end{aligned}
$$

where:

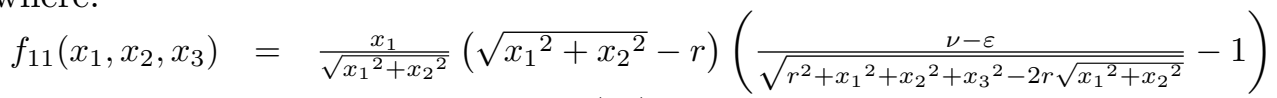

$$
\begin{aligned}
& -\quad x_{1} x_{3} \frac{\alpha}{\sqrt{x_{1}^{2}+x_{2}^{2}}}-\psi\left(x_{3}\right) x_{2} \\
& f_{12}\left(x_{1}, x_{2}, x_{3}\right)=\psi\left(x_{3}\right) x_{1}-x_{2} x_{3} \frac{\alpha}{\sqrt{x_{1}^{2}+x_{2}^{2}}}-\frac{x_{2}}{\sqrt{x_{1}^{2}+x_{2}^{2}}}\left(\sqrt{x_{1}^{2}+x_{2}^{2}}-r\right)
\end{aligned}
$$

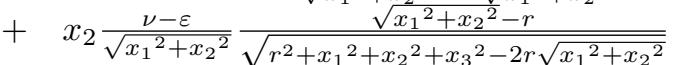

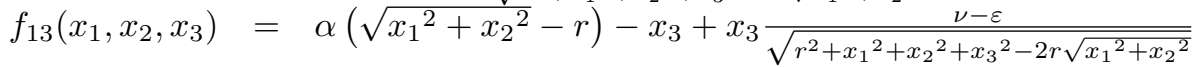

and

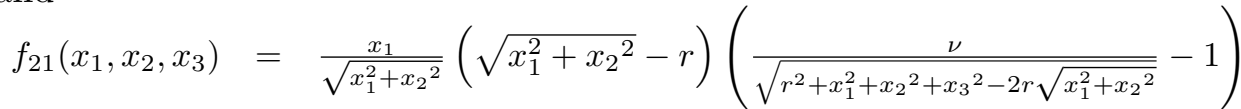

$$
\begin{aligned}
& -\quad x_{1} x_{3} \frac{\alpha}{\sqrt{x_{1}^{2}+x_{2}^{2}}}-x_{2} \gamma \\
& f_{22}\left(x_{1}, x_{2}, x_{3}\right)=x_{1} \gamma-x_{2} x_{3} \frac{\alpha}{\sqrt{x_{1}^{2}+x_{2}^{2}}}-\frac{x_{2}}{\sqrt{x_{1}^{2}+x_{2}^{2}}}\left(\sqrt{x_{1}^{2}+x_{2}^{2}}-r\right) \\
& +\quad x_{2} \frac{\nu}{\sqrt{x_{1}^{2}+x_{2}^{2}}} \frac{\sqrt{x_{1}^{2}+x_{2}^{2}}-r}{\sqrt{r^{2}+x_{1}^{2}+x_{2}^{2}+x_{3}^{2}-2 r \sqrt{x_{1}^{2}+x_{2}^{2}}}}
\end{aligned}
$$

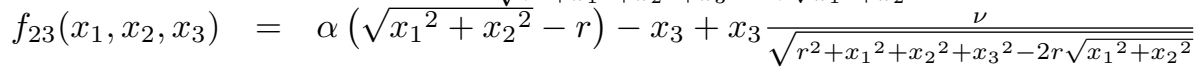

with

$$
\psi\left(x_{3}\right)=\left\{\begin{array}{lll}
\gamma & \text { if } & x_{3}>0 \\
-\gamma & \text { if } & x_{3}<0 \\
{[-\gamma, \gamma]} & \text { if } & x_{3}=0
\end{array}\right.
$$

$\alpha, \gamma$ and $\nu$ are fixed positives non nulls real numbers such that $\alpha<\gamma$ and $\varepsilon$ is a positive real value, $\varepsilon$ is small relatively to $\gamma$.

Conditions $\left.\left.\mathrm{H}_{i}\right) i=1,2,3, \mathrm{~A}_{2}\right)$ and $\left.\mathrm{H}_{2}^{1}\right)^{\prime}, \mathrm{H}_{2}^{2}$ )' are satisfied in the neighborhood of a fixed point $\bar{x}=\left(\bar{x}_{1}, 0,0\right)$, and $\bar{x}_{1}$ is a non null real value, then a sliding bifurcation of type 2 occurs. Thus simple calculus, based on the results of the previous sections and by considering also a special Poincaré section that is orthogonal to the sliding surface, gives the corresponding Poincaré map:

$$
P_{2}(x, \varepsilon)=\left\{\begin{array}{lll}
P(\varepsilon x) & \text { if } & x \geq 0 \\
P\left(\varepsilon x+\varepsilon v_{2}(x)\right)+Q \varepsilon+o\left(\varepsilon^{\frac{3}{2}}\right) & \text { if } & x<0
\end{array}\right.
$$

for any $x \in R$ and $v_{2}(x)=\frac{-x \varepsilon}{\bar{x}_{1} \gamma}$.

For all simulations, one considers the following parameters: $\alpha=20 \pi, \gamma=$ $20 \pi+0.001, \nu=0.5$ and $R=5$. So, for the first case $\varepsilon=0$, the system behavior is a limit cycle (see fig 5 ). 


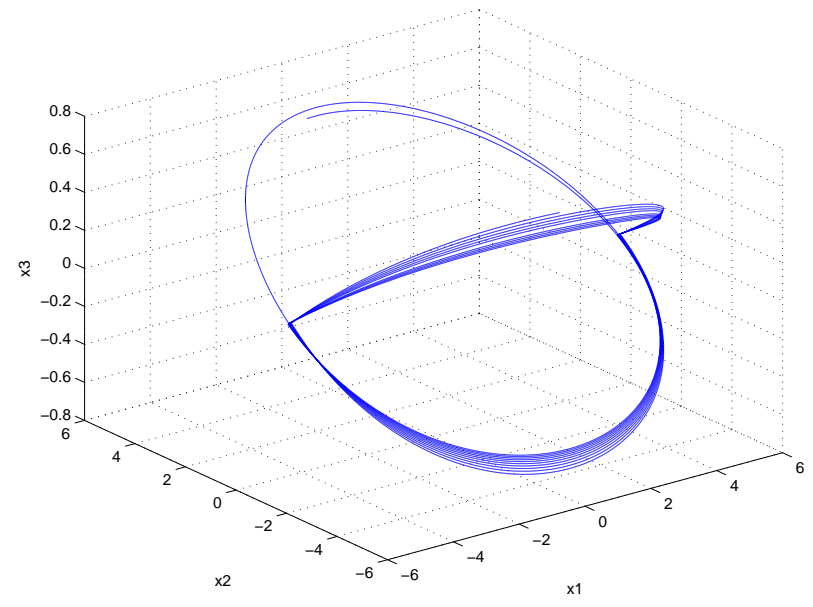

Figure 5: Limit cycle

In all the next simulations, in order to show that the system behavior converges to the limit cycle (respectively a strange attractor), one chooses the initial state $\left(x_{1}=5, x_{2}=0, x_{3}=1\right)$ outside the limit cycle (respectively a strange attractor).

According to the proposed analysis, moving the parameter $\varepsilon$ (the parameter variation can be reduced simply by a scale change), a period doubling occurs and a way to chaos is highlighted. In the figure 6 , the system behavior is given for $\varepsilon=4.5$.

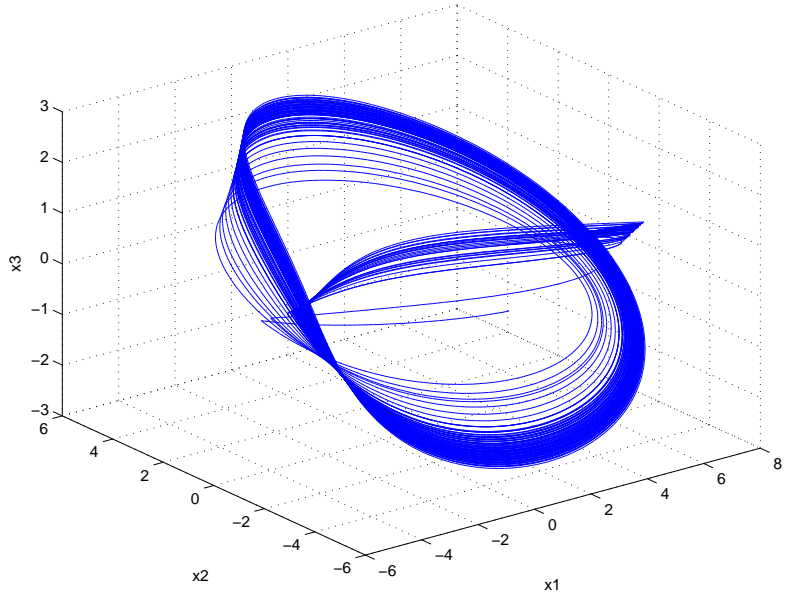

Figure 6: first steps to chaos

In the figure 7 , the system behavior is given for $\varepsilon=9.5$.

In figure 8 , a strange attractor appears for $\varepsilon=14.5$. 


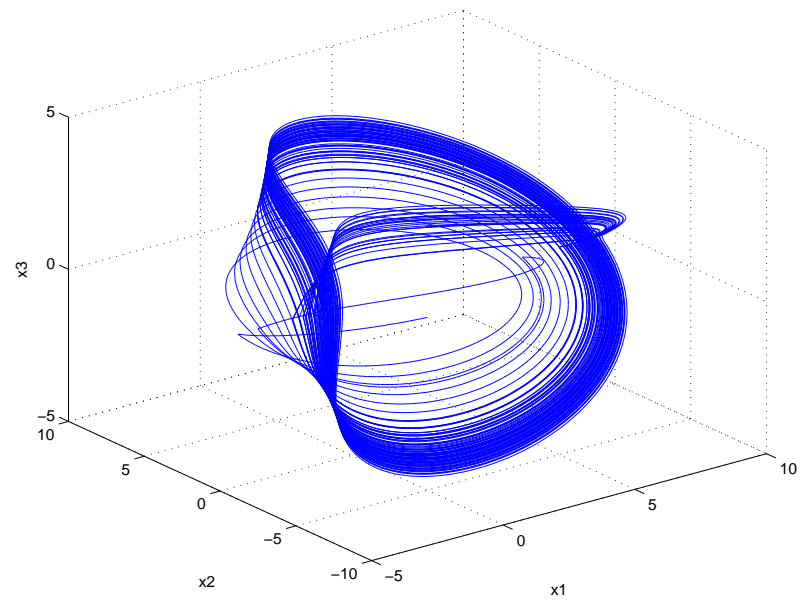

Figure 7: second steps to chaos

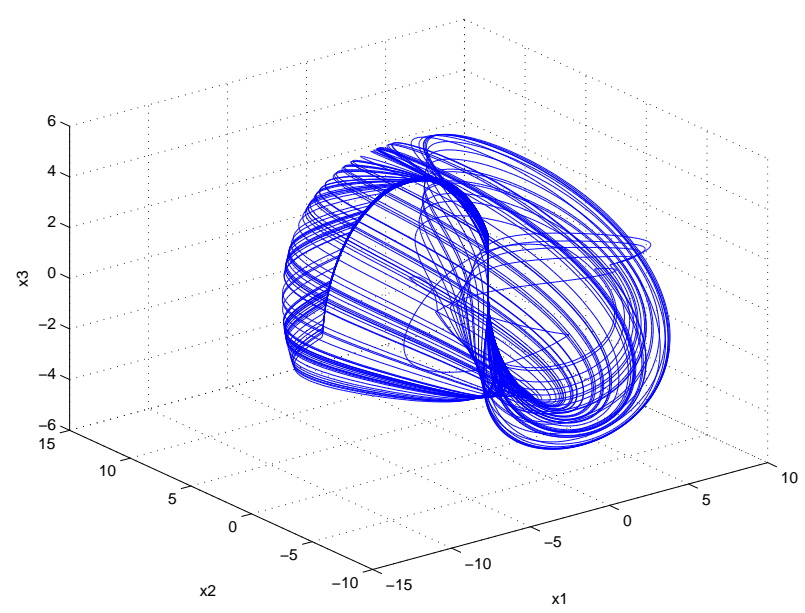

Figure 8: Chaos

\section{Conclusion}

In this paper, we have proposed a way to chaos analysis for bounded piecewise smooth systems of dimension 3 submitted to sliding bifurcations. Using the period doubling method, it is proved that chaos occurs for specific parameter variations. The obtained results can be generalized to hybrid systems such that the difference between the state dimension and the dimension of the sliding manifold is equal to two. The well-founded of this approach is highlighted by simulations results where way to chaos is explicitly shown. Their are many possible extensions of this paper in mathematical fields, for example by considering the other types of nonsmooth bifurcations or hybrid systems defined by more than two dynamics. On the other hand, some applications can be done particularly for observer design or controlling chaos, see for example [7] in power 
electronic context and [10] in private communications applications.

\section{References}

[1] D. Benmerzouk and J.P. Barbot, "Chaotic behavior analysis based on corner bifurcations", in Nonlinear Analysis: Hybrid Systems V3 N4 pp 543$550,2009$.

[2] D. Benmerzouk and J.P. Barbot, "Grazing analysis for synchronization of chaotic hybrid systems", IFAC Nolcos 2007, Pretoria, S.A., (CDROM), 2007.

[3] D. Benmerzouk and J.P. Barbot, "Lyapunov-Schmidt method dedicated to observer Analysis and Design", Mathematical problems in Engineering, Vol 2006, article ID, 43681, pp 1-28, March 2006; MPE, 2006.

[4] D. Benmerzouk and J.P. Barbot, "Observability analysis using LyapunovSchmidt method", Nolcos 2004, vol 1, pp 323-328, 2004.

[5] H. Bresis, "Analyse fonctionnelle, Théorie et Applications", Dunod, Paris, 1999.

[6] M. di Bernardo, P. Kowalczyk and A. Nordmark, "Bifurcations of dynamic systems with sliding: Derivation of normal forms", Physic D, Nonlinear Phenomena, Elseiver, volume 170, issues 3-4, pp 175-205, 2002.

[7] M. di Bernardo, C.J. Budd and A.R. Champneys, "Grazing, skipping and sliding: analysis of non-smooth dynamics of the DC/DC Buck converter", Nonlinearity II, pp 859-890, 1998.

[8] M. di Bernardo, C.J. Budd and A.R. Champneys, "Normal form maps for grazing bifurcations in n-dimensional piecewise-smooth dynamical systems", Physic D ,160, pp 222-254, 2001.

[9] M. di Bernardo, C.J. Budd, A.R. Champneys, "Unified framework for the analysis of grazing and border-collisions in piecewise smooth systems", Physical Review Letters, 86 (12), pp 2554-2556, 2001.

[10] M. Djemai, J.P. Barbot and D. Boutat, "New type of data transmission using a synchronization of chaotic systems", I.J. of Bifurcations and Chaos, Vol. 15, No. 1, pp 1-17, 2005.

[11] M.I. Feigin, "Forced oscillations in systems with discontinuous nonlinearity", Nauka, Moscow, 1994, in Russian.

[12] M.I. Feigin, "On the structure of C-bifurcation boundaries of piecewise continuous systems", PMM42, pp 820-829, 1978.

[13] A.F. Filipov, "Differential equations with discontinuous right and sides", Kluwer, Dortrecht, 1988. 
[14] P. Glendinning, "Stability, instability and chaos: An introduction to the theory of nonlinear differential equations", Cambridge University, Press, Cambridge, 1994.

[15] L.E. Nuss and J.A. Yorke, "Border-collision bifurcations for piecewise smooth one dimensional map", International Journal of bifurcation and chaos", Vol5, pp 189-207, 1995.

[16] S. Jaguste, K.M. Moudgalya, "A class of discontinuous dynamic systems, an industrial slurry high density polyethylene reactor", Chemical Engineering Science, 56, pp 3611-3621, 2001.

[17] V.I. Utkin, "Sliding modes in control optimization", Springer-Verlag, Berlin, 1992.

[18] S. Wiggins, "Introduction to applied nonlinear dynamic systems and Chaos", Texts in Applied Mathematics 2, Springer-Verlag, 1990. 